\title{
Hipotermia terapêutica em recém-nascidos com diagnóstico de encefalopatia hipóxico isquêmica: Revisão de Literatura
}

Raquel Trautenmüller Kerber Binkowski ${ }^{1}$, Angela Regina Maciel Weinmann²

\begin{abstract}
RESUMO
A Encefalopatia Hipóxico-Isquêmica é uma importante causa de dano neurológico ao nascimento, responsável por muitos óbitos e desabilidades. Até recentemente, não era passível de intervenção terapêutica; após instalada, seu tratamento resumia-se ao manejo das complicações e comorbidades, na tentativa de minimizar danos. Estudos recentes validam e recomendam a hipotermia moderada prolongada como terapêutica segura e eficaz, para a redução de mortalidade e desabilidades, em recém-nascidos com diagnóstico de Encefalopatia Hipóxico-Isquêmica. 0 objetivo desta revisão é apresentar tais estudos, analisando suas semelhanças e diferenças na abordagem terapêutica. Para tanto, foi realizada uma revisão da literatura sobre o tema, através de um levantamento bibliográfico a partir de mecanismos de busca eletrônica das bases de dados PubMed e The Cochrane Library.
\end{abstract}

Descritores: Hipóxia-Isquemia Encefálica; Hipotermia Induzida; Recém-nascido.

\section{Hypothermia for neonatal hypoxic ischemic encephalopathy: Literature Review}

\begin{abstract}
Neonatal Hypoxic Ischemic Encephalopathy is an important cause of neurological damage at birth, responsible for many deaths and disabilities. Until recently there was no therapeutic option to this condition; after installed its treatment summed up the management of complications and comorbidities in an attempt to minimize damage. Recent studies validate and recommend prolonged moderate hypothermia as a safe and effective therapy, reducing mortality and disabilities in newborns diagnosed with Hypoxic Ischemic Encephalopathy. The aim of this review is to present such studies, analyzing the similarities and differences in therapeutic approach. For this we conducted a literature review by searching in databases PubMed and The Cochrane Library.
\end{abstract}

Descriptors: Hypoxia-Ischemia, Brain; Hypothermia, Induced; Newborn.

${ }^{1}$ Médica Neuropediatra pela Universidade Federal de Santa Maria (UFSM), Santa Maria, RS, Brasil.

${ }^{2}$ Médica Neonatologista pela Universidade Federal de Santa Maria (UFSM), Santa Maria, RS, Brasil. 


\section{Introdução}

A asfixia perinatal é um agravo ao feto ou ao recém-nascido $(\mathrm{RN})$ que ocorre com maior frequência nos períodos pré e intraparto, caracterizado por privação de oxigênio (hipóxia) e distúrbio perfusional (isquemia), com repercussões sistêmicas múltiplas. Dentre os órgãos e/ou sistemas afetados pela asfixia destaca-se o sistema nervoso central, cujo envolvimento configura a chamada Encefalopatia Hipóxico-Isquêmica (EHI) ${ }^{1}$.

A EHI é a manifestação clínica da asfixia perinatal mais estudada e descrita na literatura, cujos sintomas, embora inespecíficos, envolvem, com frequência, a presença de convulsões, geralmente prolongadas e resistentes ao uso de fármacos antiepilépticos (FAE). De acordo com a gravidade do quadro são definidos 3 estágios clínicos, conforme 0 sistema de classificação de $\mathrm{EHI}^{1,2,3}$. No estágio $10 \mathrm{RN}$ apresenta-se hiperalerta, com tremores grosseiros de extremidades, hiperatividade dos reflexos miotáticos, baixo limiar para o reflexo de Moro e tono muscular preservado. Nesse estágio, o quadro clínico se resolve dentro da primeira semana, sem quaisquer medidas específicas. Algumas crianças podem persistir com hiperexcitabilidade por períodos maiores. No estágio 2, o RN apresenta-se letárgico, com discreta hipotonia, hipoatividade ou abolição dos reflexos arcaicos, podendo apresentar crises convulsivas. Nesse estágio predomina a atividade parassimpática, com tendência à bradicardia e constrição pupilar. Pode ocorrer melhora ou comprometimento progressivo do estado de consciência. Normalmente o quadro clínico se mantém por mais de 14 dias, habitualmente ocorrendo alguma melhora ao final da primeira semana. 0 estágio 3 se caracteriza por coma com hipotonia global ou posturas anormais, ausência de reflexos arcaicos e miotáticos e alterações da motricidade ocular extrínseca e intrínseca. Nessa fase, pode haver alguma melhora do estado de consciência entre 12 e 24 horas após o parto, porém muitos neonatos continuam comatosos com progressão das convulsões para status epilepticus. Podem evoluir para o óbito e, entre os sobreviventes, ocorrem sequelas neurológicas relevantes.

Em países desenvolvidos, a asfixia neonatal afeta 3-5:1.000 nascidos vivos, com subsequente EHI moderada ou severa em 0,5-1:1.000. Trata-se de um dos maiores problemas mundiais, provocando óbito em 10-60\% das crianças afetadas, sendo que no mínimo $25 \%$ dos sobreviventes apresentam sequelas neurológicas a longo prazo 4 . Dentre os com encefalopatia severa, $60 \%$ morrem, e a maioria, senão todos os sobreviventes, tornam-se portadores de deficiências ${ }^{5}$.

Já nos países em desenvolvimento, a asfixia tem sido relatada em $9,7 \%$ das gestações de alto risco, com uma incidência de $31 \%$ de EHI na presença de asfixia perinatal1. No Brasil, é a segunda causa de mortalidade neonatal, tanto no primeiro dia quanto na primeira semana de vida, sendo responsável por 18,3\% e 16,7\% dos óbitos nesses períodos, respectivamente ${ }^{6}$. A EHI é a causa mais frequente envolvida na etiopatogenia da paralisia cerebral, uma encefalopatia crônica infantil caracterizada por distúrbios motores de caráter não progressivo, levando a distúrbios de motricidade, tônus e postura, podendo ou não se associar à déficits cognitivos e sensoriais. Nos países em desenvolvimento, considerandose todos os níveis de paralisia cerebral, a incidência estimada é de 7:1.000 nascidos vivos¹.

Embora a EHI seja uma importante causa de desabilidade e esteja associada à alta mortalidade no período neonatal, até recentemente, não era passível de intervenção terapêutica. Após instalada, a equipe assistente apenas poderia tratar as complicações e comorbidades, na tentativa de minimizar danos. No entanto, estudos clínicos em crianças com evidência de hipóxia intraparto e encefalopatia moderada a severa demonstraram um modelo bifásico de morte neuronal. Esses estudos evidenciaram a presença de metabolismo cerebral oxidativo normal imediatamente após o nascimento, seguido por uma "falência energética secundária", cujo grau está diretamente ligado ao desfecho (mortalidade e desenvolvimento neurológico $)^{7,8}$. Portanto, está demonstrado que existe uma "janela de oportunidade", que corresponde ao intervalo de tempo entre a reanimação em sala de parto de um RN asfixiado e a fase secundária de alteração no metabolismo e lesão cerebral. Baseado na comprovação da existência desse período, passível de intervenção e do efeito sabidamente neuroprotetor da hipotermia ${ }^{9,10}$, vários grupos de pesquisa se dedicaram ao estudo da hipotermia moderada prolongada como uma terapêutica promissora em RN com diagnóstico de EHI.

Muitas dúvidas acerca da segurança e da efetividade da hipotermia no tratamento da EHI no período neonatal foram levantadas nos últimos anos. No entanto, diversos estudos mostraram que a mesma reduziu a ocorrência de desfechos desfavoráveis (morte e desabilidade) no RN, ao mesmo tempo que aumentou o número de sobreviventes saudáveis, sem causar efeitos adversos $4,5,11,12,13,14,15$.

Frente a este contexto, o objetivo do presente artigo foi revisar e apresentar estudos sobre a terapia com hipotermia em recém-nascidos com diagnóstico de Encefalopatia Hipóxico-Isquêmica, analisando suas semelhanças e diferenças na abordagem terapêutica, a partir de revisão da literatura nas bases de dados PubMed e The Cochrane Library. 


\section{Fisiopatologia da EHI}

Estudos clínicos e experimentais demonstram que a morte neuronal ocorre em duas fases após um dano hipóxicoisquêmico global ${ }^{16,17,18}$. Se 0 insulto é severo, há imediatamente uma "morte neuronal primária", relacionada à hipóxia celular com exaustão dos estoques de energia celular ("primeira falha energética"). Após um período latente de pelo menos seis horas, inicia-se a chamada fase secundária de "morte celular tardia"19. Nesta fase, os mecanismos envolvidos na morte neuronal incluem edema citotóxico, falência mitocondrial, acúmulo de toxinas, ativação de morte celular (análoga ao desenvolvimento de apoptose), síntese de óxido nítrico, danos provocados por radicais livres e ação citotóxica por ativação da micróglia ${ }^{20}$. A fase tardia é associada com encefalopatia e aumenta a atividade epileptogênica, sendo responsável por uma proporção significante do total de células perdidas após insultos muito severos ${ }^{4,19}$.

Em crianças com evidência de hipóxia intraparto e encefalopatia moderada a severa, estudos de ressonância magnética são consistentes com esse modelo bifásico de morte neuronal. Esses estudos demonstraram metabolismo cerebral oxidativo normal imediatamente após o nascimento, seguido por uma "falência energética secundária", cujo grau pode ser considerado um fator preditor para o desfecho final (mortalidade e desenvolvimento neurológico)7,8.

Existem mecanismos já postulados, segundo os quais a hipotermia é neuroprotetora. A hipotermia modifica as células programadas para apoptose, levando-as à sobrevida ${ }^{21}$. Em porcos recém-nascidos, 12 horas de hipotermia leve após a reanimação reduziu significativamente o número de células apoptóticas, mas não o número de células necróticas ${ }^{9}$. Além disso, a hipotermia protege os neurônios através da redução da taxa metabólica cerebral, atenuando a liberação de aminoácidos excitatórios (glutamato, dopamina), melhorando o dano isquêmico pela absorção de glutamato e diminuindo a produção de óxido nítrico e radicais livres ${ }^{10}$.

Vários modelos experimentais com animais a termo e pré-termo demonstraram que a redução da temperatura cerebral em 2 a $3^{\circ} \mathrm{C}$ imediatamente após um insulto hipóxico-isquêmico reduz o gasto energético, melhorando subsequentemente testes de performance e/ou reduzindo a perda histológica neuronal ${ }^{22,23,24}$. Modelos de cálculo de temperatura sugerem que a diminuição da temperatura central dos recém-nascidos (RNs) abaixo de $35^{\circ} \mathrm{C}$ é necessária para produzir alguma redução de temperatura em estruturas cerebrais profundas ${ }^{25}$.

A partir dessas constatações, várias publicações surgiram no intuito de reproduzir tais efeitos em RNs.

\section{Metodologia}

A presente revisão bibliográfica foi realizada com base em publicações do tipo ensaio clínico randomizado e meta-análise nas bases de dados The Cochrane Library e PubMed. A procura foi realizada buscando-se por "Título, resumo ou palavra-chave":

1) "hypoxic ischaemic encephalopathy". Foram encontradas nove revisões da Cochrane, dentre elas uma pertinente à busca: "Cooling for newborns with hypoxic ischaemic encephalopathy". Também foram encontradas duas "outras revisões", sendo uma delas pertinente: "Neurological outcomes at 18 months of age after moderate hypothermia for perinatal hypoxic ischaemic encephalopathy: synthesis and meta-analysis of trial data"11.

2) "hypoxic ischemic encephalopathy". Foram encontradas seis "outras revisões", dentre elas três pertinentes à busca, sendo selecionada a mais recente: "Hypothermia for neonatal hypoxic ischemic encephalopathy: an updated systematic review and meta-analysis"13.

A partir destas revisões e meta-análises, utilizou-se o PubMed como ferramenta de busca para os estudos citados. Foram incluídos na análise três ensaios clínicos randomizados ${ }^{26,5,27}$ com forte qualidade metodológica e grande número de pacientes. Além disso, foi adicionado um ensaio clínico randomizado ${ }^{12} \mathrm{com}$ adequado método científico que, embora tenha sido interrompido devido ao acúmulo de evidências a favor do tratamento, realizava um método muito simples e prático de hipotermia. Esta busca bibliográfica teve início em maio de 2011, sendo periodicamente repetida até janeiro de 2013. 


\section{Resultados e discussão}

Ensaios clínicos randomizados

\section{Cool-Cap Trial $^{26}$}

Trata-se do primeiro ensaio clínico com um número significativo de crianças. Este estudo incluiu 25 centros em 4 países (Nova Zelândia, Inglaterra, Estados Unidos e Canadá), totalizando 234 pacientes entre julho de 1999 a janeiro de 2002.

Os critérios de inclusão utilizados foram evidência de asfixia periparto, definida como: Apgar menor ou igual a 5 no décimo minuto de vida, necessidade de reanimação até pelo menos o décimo minuto de vida, ou acidose severa $(\mathrm{pH}<$ 7,0 ou $\mathrm{BE} \leq-16 \mathrm{mmol} / \mathrm{L}$ ) dentro da primeira hora após o nascimento. Adicionalmente, deveriam apresentar encefalopatia moderada ou severa (definida pelos critérios de Sarnat) ou evidências clínicas de convulsão, bem como alterações ao eletroencefalograma (EEG - voltagem moderada ou severamente anormal, convulsões).

O procedimento proposto consistia em resfriar a cabeça do RN através do uso de um equipamento (Olympic Medical Cool Care System) composto por um capacete no qual circulava água com temperatura entre 8 e $12^{\circ} \mathrm{C}$. Concomitantemente, os pacientes eram mantidos em berço aquecido, sendo monitorizada a temperatura retal.

Os pacientes do grupo controle recebiam os cuidados-padrão de todo recém-nascido admitido em uma Unidade de Terapia Intensiva (UTI) neonatal, ou seja, eram mantidos em berço aquecido, com temperatura retal em torno de 36,8 a $37,2^{\circ} \mathrm{C}$.

Avaliaram-se as seguintes complicações, nos dois grupos, durante os primeiros 7 dias de vida: arritmia cardíaca, trombose venosa, hipotensão, coagulopatia, alteração de função renal e/ou hepática, distúrbios hidroeletrolíticos, alterações hematológicas, acidose metabólica, necessidade de suporte ventilatório, infecção, hipoglicemia, dificuldade no controle térmico e alterações de pele. Não houve diferença estatisticamente significante, entre os grupos, quanto a essas complicações.

Os desfechos analisados foram divididos em:

- Primários: mortalidade e desabilidade severa aos 18 meses. Como desabilidade severa foram considerados: Escala "Gross Motor Function" (GMF) entre 3 e 5, Escala "Bayley mental developmental index" (MDI) inferior a 70 ou dano visual cortical bilateral.

- Secundários: disfunção de múltiplos órgãos (pelo menos 3), epilepsia, desabilidades múltiplas (pelo menos duas das seguintes: GMF entre 3 e 5; MDI inferior a 70, epilepsia, dano visual cortical bilateral ou perda auditiva neurossensorial).

Ao avaliar tais desfechos, concluiu-se que a hipotermia não foi protetora naquelas crianças com alterações severas ao EEG, possivelmente pela gravidade da injúria. Porém, nos RNs com alterações menos relevantes ao EEG, houve melhora da sobrevida sem desabilidades severas, demonstrada por uma redução maior que $50 \%$ em tais desabilidades aos 18 meses e de um melhor desempenho na Escala MDI.

\section{$\underline{\text { NICHD Trial }}^{5}$}

O ensaio conduzido pelo National Institute of Child Health and Human Development foi publicado alguns meses após o estudo anterior. Realizado em 15 centros nos Estados Unidos entre julho de 2000 e maio de 2003, contemplou 208 RNs.

Neste ensaio, a acidose severa, tal como definida no Cool-Cap Trial, era considerada critério de inclusão na evidência de asfixia periparto. Porém, caso a gasometria demonstrasse alterações limítrofes (pH entre 7,01 e 7,15 ou BE entre -10 e - $15,9 \mathrm{mmol} / \mathrm{L}$ ) ou não houvesse amostra de sangue disponível, critérios adicionais eram requeridos para definir asfixia: evidência de complicações perinatais (p.ex., prolapso de cordão), além de Apgar menor ou igual a 5 no décimo minuto de vida ou necessidade de reanimação até pelo menos o décimo minuto de vida.

Assim como no estudo anterior, os RNs deveriam apresentar encefalopatia moderada ou severa (definida pelos critérios de Sarnat) ou evidências clínicas de convulsão. Não foram utilizados critérios eletroencefalográficos.

$\mathrm{O}$ procedimento visava manter uma temperatura esofágica em torno de $33,5^{\circ} \mathrm{C}$ utilizando um cobertor (Blanketrol II Hyper-Hypothermia System, Cincinnati Sub-Zero) no qual circulava água, propiciando o resfriamento. O RN ficava envolvido nesse cobertor de forma a realizar hipotermia corporal total. Os pacientes do grupo controle recebiam os cuidados-padrão.

Foram avaliadas as seguintes complicações nos dois grupos durante 0 procedimento: arritmia cardíaca, acidose metabólica, sangramento, alterações de pele e taxa de mortalidade. Durante o período de internação, foram comparadas as prevalências de: hipotensão, arritmia cardíaca, coagulopatia, alteração de função renal e/ou hepática, distúrbios hidroeletrolíticos, necessidade de suporte ventilatório, infecção e hipoglicemia. Também foram avaliadas, por ocasião da alta, a modalidade de alimentação e o uso de FAE. Não houve diferença estatisticamente significante quanto a essas complicações nos dois grupos. 
Os desfechos primários analisados foram mortalidade e desabilidade severa aos 18 e 22 meses. Quanto ao grau de desabilidade, foram divididos em dois grupos: severa (Escala GMF entre 3 e 5, Escala MDI inferior a 70, surdez ou cegueira) e moderada (Escala GMF: 2, Escala MDI entre 70 e 84, déficit auditivo ou epilepsia).

Ao avaliar tais desfechos, concluiu-se que a hipotermia reduziu o risco de mortalidade ou desabilidade em todos os RNs com EHI, seja ela moderada ou severa. Ao contrário do Cool Cap Trial, os critérios de elegibilidade eram facilmente realizados, não sendo necessária a disponibilidade de um aparelho de EEG.

\section{TOBY Trial $^{27}$}

O Total Body Hypothermia for Neonatal Encephalopathy constitui-se no maior ensaio clínico que estudou a hipotermia em RNs. Incluía 42 centros em 5 países (Inglaterra, Israel, Finlândia, Hungria e Suécia). No período de dezembro de 2002 a novembro de 2006 foram avaliados 325 pacientes.

A hipotermia era induzida utilizando um cobertor (Tecotherm TS 200, Tec-Com) semelhante ao utilizado no NICHD Trial. Os pacientes do grupo controle também recebiam os cuidados-padrão.

Foram avaliadas as seguintes complicações nos dois grupos durante o período de internação: arritmia cardíaca, trombose venosa, hipotensão, coagulopatia, sangramentos, hipertensão pulmonar, alteração de função renal, alterações hematológicas, necessidade de suporte ventilatório, infecção, enterocolite necrotizante. Os efeitos adversos foram similares nos dois grupos, sendo que os efeitos adversos sérios não foram associados à hipotermia.

Os desfechos analisados foram divididos em:

- Primários: mortalidade e desabilidade severa aos 18 meses. Para caracterizar desabilidade severa foram considerados os mesmos critérios utilizados no Cool Cap Trial.

- Secundários: mortalidade e desabilidade severa aos 18 meses (mesmos do desfecho primário), porém considerando outras alterações além daquelas descritas como desabilidade severa: paralisia cerebral, déficit auditivo, convulsões necessitando uso de FAE e microcefalia. Também avaliou a sobrevida sem anormalidades neurológicas, definida como: Escala GMF: 1, Escala MDI superior a 84, visão e audição normais.

Ao avaliar tais desfechos, concluiu-se que a hipotermia não reduziu significativamente a combinação mortalidadedesabilidade, mas resultou em melhora dos desfechos neurológicos nos sobreviventes.

\section{$\underline{\text { ICE Trial }}^{12}$}

Este estudo incluiu 28 centros em 4 países (Austrália, Nova Zelândia, Canadá e Estados Unidos), avaliando 221 pacientes entre fevereiro de 2001 a julho de 2007.

Os critérios de asfixia utilizados foram os mesmos utilizados no Cool-Cap Trial, com exceção do limiar considerado no $B E$ (utilizado $B E \leq-12 \mathrm{mmol} / \mathrm{L}$ ) e a idade gestacional (IG) - incluídos RNs com IG $\geq 35$ semanas. Além disso, os RNs deveriam também apresentar encefalopatia moderada ou severa (definida pelos critérios de Sarnat) ou evidências clínicas de convulsão. Este foi o único ensaio clínico, entre os aqui descritos, a incluir crianças com IG entre 35 e 36 semanas.

Esse ensaio clínico foi único, pois utilizou um método muito simples e prático para realizar a terapêutica: consistia em desligar o berço aquecido e, conforme necessidade de maior resfriamento, aplicar pás de gel refrigeradas no tórax, cabeça e ombros. Conforme fosse atingida a temperatura ideal, essas pás eram removidas - ou recolocadas, se necessário. Tal procedimento já era utilizado nos "times de transporte", uma vez que muitos desses bebês nasciam em centros distantes daqueles onde havia uma UTI neonatal disponível; portanto, necessitavam iniciar a hipotermia já durante o transporte, a fim de evitar a exclusão pela admissão após 6 horas de vida. Os pacientes do grupo controle eram mantidos conforme os cuidados-padrão.

Foram avaliadas as seguintes complicações nos dois grupos durante o período de internação: arritmia cardíaca, hipotensão, trombose venosa, coagulopatia, sangramentos, alteração de função renal e/ou hepática, alterações hematológicas, necessidade de suporte ventilatório, infecção, enterocolite necrotizante e mortalidade. Os efeitos adversos foram similares nos dois grupos.

Os desfechos analisados foram divididos em:

- Primários: mortalidade e desabilidade severa aos 24 meses. Como desabilidade severa foram considerados: Escala MDI de pelo menos 2 desvios-padrão (DP) abaixo da média; Escala GMF entre 2 e 5; presença de paralisia cerebral, cegueira ou deficiência auditiva.

- Secundários: mortalidade e desabilidade severa (e seus componentes individuais) aos 24 meses, bem como sobrevida sem desabilidade. Os componentes individuais de desabilidade severa foram os seguintes: atraso no desenvolvimento 
neuropsicomotor, cegueira e deficiência auditiva. Definiu-se como sobrevida sem desabilidade a presença de Escala GMF: 0 e Escala MDI até 1 DP abaixo da média, ausência de cegueira e/ou surdez.

Ao avaliar tais desfechos, concluiu-se que a hipotermia não reduziu significativamente a combinação desabilidade severa e seus componentes individuais, porém houve uma diminuição significante na mortalidade e houve um aumento da sobrevida livre de desabilidade.

Convém enfatizar que esse estudo planejava recrutar $300 \mathrm{RNs}$, no entanto, precisou ser interrompido devido ao acúmulo de evidências, naquele momento, a favor da hipotermia, levando os pesquisadores à responsabilidade ética de fornecer tratamento a todas as crianças.

Nas tabelas 1 e 2 encontram-se as características desses estudos.

Tabela 1 - Características dos ensaios clínicos

\begin{tabular}{c|c|c|c|c}
\hline Estudo (Autor, Ano) & Hipotermia: Controles (n) & Método & $\begin{array}{c}\text { Temperatura } \\
\text { central alvo }\left({ }^{\circ} \mathrm{C}\right)\end{array}$ & Duração (horas) \\
\hline $\begin{array}{c}\text { Cool-Cap Trial } \\
\text { (Gluckman et al, 2005) }\end{array}$ & $116: 118$ & Seletiva & $34-35$ & 72 \\
\hline $\begin{array}{c}\text { NICHD Trial (Shankaran et al, } \\
\text { 2005) }\end{array}$ & $102: 106$ & Total & 33,5 & 72 \\
\hline TOBY Trial (Azzopardi et al, & $163: 162$ & & $33-34$ & 72 \\
\hline 2009) & $110: 111$ & Total & & 72 \\
\hline
\end{tabular}

Tabela 2 - Características dos ensaios clínicos

\begin{tabular}{|c|c|c|c|c|}
\hline Estudo & Critérios de inclusão & $\begin{array}{l}\text { Critérios de } \\
\text { exclusão }\end{array}$ & Desfecho primário & Desfecho secundário \\
\hline $\begin{array}{c}\text { Cool-Cap } \\
\text { Trial }\end{array}$ & $\begin{array}{c}\text { - evidência de asfixia periparto } \\
\text { - EHI moderada/ severa ou } \\
\text { convulsão } \\
\text { - alterações } \\
\text { ao EEG } \\
\text { - IG } \geq 36 \text { sem. }\end{array}$ & $\begin{array}{c}>5,5 \text { horas de } \\
\text { vida, alta dose de } \\
\text { FAE, alterações } \\
\text { congênitas } \\
\text { maiores, TCE } \\
\text { grave, peso } \\
<1.800 \mathrm{~g}, \\
\text { microcefalia, } \\
\text { estatura > } 2 \mathrm{DP}, \\
\text { pacientes críticos }\end{array}$ & $\begin{array}{l}\text { mortalidade e } \\
\text { desabilidade severa } \\
\text { aos } 18 \text { meses }\end{array}$ & $\begin{array}{l}\text { disfunção de múltiplos órgãos } \\
\text { (pelo menos 3), epilepsia, } \\
\text { desabilidades múltiplas }\end{array}$ \\
\hline $\begin{array}{c}\text { NICHD } \\
\text { Trial }\end{array}$ & $\begin{array}{c}\text { - evidência de asfixia periparto } \\
\text { - EHI } \\
\text { moderada/ severa ou } \\
\text { convulsão } \\
\text { - IG } \geq 36 \text { sem. }\end{array}$ & $\begin{array}{c}>6 \text { horas de } \\
\text { vida, alterações } \\
\text { congênitas } \\
\text { maiores, CIUR } \\
\text { severa, pacientes } \\
\text { críticos }\end{array}$ & $\begin{array}{c}\text { mortalidade e } \\
\text { desabilidade severa } \\
\text { aos } 18 \text { e } 22 \text { meses }\end{array}$ & \\
\hline
\end{tabular}




\begin{tabular}{|c|c|c|c|c|}
\hline $\begin{array}{c}\text { TOBY } \\
\text { Trial }\end{array}$ & $\begin{array}{c}\text { - evidência de asfixia periparto } \\
\text { - EHI } \\
\text { moderada / severa ou } \\
\text { convulsão + } \\
\text { - alterações ao EEG } \\
\text { - IG } \geq 36 \text { sem. }\end{array}$ & $\begin{array}{l}>6 \text { horas de } \\
\text { vida, alterações } \\
\text { congênitas } \\
\text { maiores }\end{array}$ & $\begin{array}{c}\text { mortalidade e } \\
\text { desabilidade severa } \\
\text { aos } 18 \text { meses }\end{array}$ & $\begin{array}{c}\text { mortalidade e desabilidade } \\
\text { severa (incluindo outras } \\
\text { alterações) aos } 18 \text { meses, } \\
\text { sobrevida sem anormalidades } \\
\text { neurológicas }\end{array}$ \\
\hline $\begin{array}{l}\text { ICE } \\
\text { Trial }\end{array}$ & $\begin{array}{c}\text { - evidência de asfixia periparto } \\
\text { - EHI } \\
\text { moderada / severa ou } \\
\text { convulsão } \\
\text { - IG } \geq 35 \text { sem. }\end{array}$ & $\begin{array}{c}>6 \text { horas de vida, } \\
\text { peso }<2.000 \mathrm{~g}, \\
\text { anormalidades } \\
\text { congênitas } \\
\text { maiores, } \\
\text { sangramento, } \\
\text { necessidade de } \\
\mathrm{FiO}_{2}>80 \% \text {, morte } \\
\text { iminente }\end{array}$ & $\begin{array}{c}\text { mortalidade e } \\
\text { desabilidade severa } \\
\text { aos } 24 \text { meses }\end{array}$ & $\begin{array}{l}\text { mortalidade e desabilidade } \\
\text { severa (e seus componentes } \\
\text { individuais) aos } 24 \text { meses, } \\
\text { sobrevida sem desabilidade }\end{array}$ \\
\hline
\end{tabular}

TCE: trauma craniencefálico; CIUR: restrição de crescimento intrauterino.

\section{$\underline{\text { Revisões sistemáticas e meta-análises }}$}

\section{Cooling for newborns with hypoxic ischaemic encephalopathy ${ }^{4}$}

Na tentativa de reunir a literatura disponível até então sobre o tratamento com hipotermia em RNs com EHI, em 2007 foi publicada uma revisão sistemática, que foi revisada em 2013. O objetivo principal dessa revisão foi o de avaliar os desfechos e efeitos adversos da hipotermia terapêutica. Foram incluídos oito ensaios clínicos randomizados; desses, seis eram estudos-piloto e os outros dois foram o Cool-Cap Trial e o NICHD Trial. Quatro estudos utilizaram resfriamento da cabeça, enquanto os demais aplicaram resfriamento corporal total. O período de hipotermia foi de 72 horas, exceto em um que realizou o procedimento por um período de 48 horas.

Os critérios de inclusão foram a evidência de asfixia periparto associada à EHI, segundo os critérios de Sarnat, bem como ausência de anormalidades congênitas maiores reconhecíveis ao nascimento.

Os desfechos foram divididos em:

- Primários: mortalidade; atraso ou desabilidade maior no desenvolvimento neuropsicomotor; presença de paralisia cerebral, cegueira ou deficiência auditiva.

- Secundários: efeitos adversos da terapia (arritmia cardíaca, alteração de função renal, hipotensão, alterações hemograma e/ou ionograma, sepse), severidade da encefalopatia, alterações no EEG e/ou na ressonância magnética, convulsões, número de dias para iniciar a alimentação por via oral.

Do ponto de vista metodológico, de maneira geral, houve consistência entre os oito estudos, que analisaram 638 crianças. Isso foi particularmente verdadeiro para os dois maiores, o Cool-Cap e NICHD Trial, justamente os que mais pesaram na análise dos resultados. Após análise, concluiu-se que a hipotermia terapêutica proporcionou redução da mortalidade e das desabilidades aos 18 meses, com uma redução do risco relativo de $24 \%$ e do risco absoluto de $15 \%$, com um NNT de 7 .

Esta análise também evidenciou um aumento da trombocitopenia e hipotensão devido à hipotermia, no entanto, os benefícios em termos de sobrevida e desenvolvimento neurológico suplantaram os efeitos adversos a curto prazo.

Os autores da revisão, entretanto, fazem uma ressalva: embora os resultados encontrados fossem promissores, havia ainda, no momento da publicação, outros ensaios clínicos em andamento. Portanto, aguardar tais resultados seria de grande valor, uma vez que poderiam vir a contribuir com novas informações a respeito da efetividade e a segurança da hipotermia.

Neurological outcomes at 18 months of age after moderate hypothermia for perinatal hypoxic ischaemic encephalopathy: synthesis and meta-analysis of trial data ${ }^{11}$

A revisão anterior, como os próprios autores enfatizaram, mostrou dados encorajadores a favor da hipotermia, mas a comunidade científica ainda aguardava os dados do TOBY Trial. Quando esse foi publicado, dispunha-se do terceiro grande ensaio clínico mostrando efeito benéfico da hipotermia. Entretanto, tais benefícios diferiam entre os estudos, bem como sua significância, o que tornou necessário reunir todos os dados dos ensaios anteriores e analisá-los conjuntamente, para chegar 
a uma conclusão definitiva. Inclusive porque, conforme visto anteriormente, existiam pequenas diferenças entre os estudos.

Assim, em 2010, foi publicada a metanálise que reuniu o Cool Cap Trial, o NICHD Trial e o TOBY Trial, totalizando 767 crianças. Foram incluídos também outros 7 estudos, cuja informação sobre mortalidade era adequada, porém sem informações sobre o desenvolvimento neurológico, totalizando 1.320 crianças. Avaliando os três maiores ensaios clínicos, observou-se a semelhança nos critérios de inclusão.

Os desfechos, referentes à desabilidade, foram definidos do mesmo modo no Cool Cap Trial e no TOBY Trial: Escala MDI inferior a 70 ou Escala GMF entre 3 e 5 ou cegueira cortical bilateral. No NICHD Trial, foram definidos como Escala MDI entre 70 e 84 e, adicionalmente, Escala GMF: 2 ou perda auditiva ou epilepsia. Individualmente, todos mostraram redução da mortalidade e da desabilidade, porém, essa redução foi estatisticamente significante somente no NICHD Trial.

Assim, para analisar conjuntamente os dados de tais estudos, os desfechos foram divididos em:

- Primários: mortalidade e desabilidade aos 18 meses. Houve redução significativa na combinação entre ambos: 19\% no risco relativo (RR 0.81, a: $95 \%$, intervalo de confiança de 0.71 a 0.93, $p=0.002$ ) e $11 \%$ no risco absoluto (RA -0.11 , $a$ : 95\%, intervalo de confiança de -0.18 a -0.04) com NNT: 9 (a: $95 \%$, intervalo de confiança de 5 a 25). Avaliando somente 0 quesito mortalidade (dez estudos), também houve redução significativa: $22 \%$ no risco relativo (RR 0.78, a: $95 \%$, intervalo de confiança de 0.66 a $0.93, p=0.005$ ) e $7 \%$ no risco absoluto (RA -0.07 , $a: 95 \%$, intervalo de confiança de -0.12 a -0.02 ) com NNT: 14 (a: 95\%, intervalo de confiança de 8 a 47).

- Secundários: sobrevida com função neurológica normal, definida como Escala MDI superior a 84, visão e audição normais. Houve um aumento significativo no número de RNs normais: $53 \%$ no risco relativo (RR 1.53, a: $95 \%$, intervalo de confiança de 1.22 a 1.93, p < 0.001) e 12\% no risco absoluto (RA 0.12, a: 95\%, intervalo de confiança de 0.06 a 0.18 ) com NNT: 8 (a: 95\%, intervalo de confiança de 5 a 17). Também houve, entre os sobreviventes, redução das taxas de desabilidade severa $(p=0.006)$, paralisia cerebral $(p=0,004)$, Escala MDI inferior a $70(p=0.01$ para o escore mental e $p=0.02$ para o escore psicomotor).

Como os estudos incluídos eram de alta qualidade metodológica, a meta-análise permitiu concluir que há alto nível de evidência de eficácia da hipotermia na EHI, com efeito terapêutico significante (redução da mortalidade e desabilidade aos 18 meses). Portanto, concluiu-se que as mínimas diferenças entre os ensaios clínicos sugerem que a implementação da terapêutica depende mais de uma equipe bem treinada do que do método de hipotermia utilizado.

\section{Hypothermia for Neonatal Hypoxic Ischemic Encephalopathy: An Updated Systematic Review and Meta-analysis ${ }^{13}$}

Apesar de toda evidência acumulada, a revisão anterior ainda deixou algumas dúvidas. Havia clara evidência de benefício significante na EHI moderada; no entanto, na EHI grave, embora também houvesse melhora, esta não era significante. Entretanto, tal revisão não contemplava 3 ensaios clínicos recentes ${ }^{12,14,15}$ o que tornou relevante a publicação da presente meta-análise em 2012, que totalizou 1.214 pacientes.

Todos os pacientes incluídos tinham menos de 6 horas de vida e apresentavam evidência de asfixia neonatal associada à encefalopatia moderada ou severa, além de IG $\geq 36$ semanas (exceto o ICE Trial). Quanto ao método, quatro utilizaram hipotermia corporal total e três hipotermia seletiva, por 72 horas.

Foram utilizados, para definir desabilidades: diagnóstico de paralisia cerebral, atraso no desenvolvimento (de pelo menos 2 DP abaixo da média nas seguintes escalas: MDI; combinação Bayley III, avaliação de Griffiths e quociente de Brunet-Lézine; Escala de desenvolvimento de Gesell Child; Teste de quociente de inteligência), cegueira e déficit auditivo.

Assim, para analisar conjuntamente os dados de tais estudos, os desfechos foram divididos em:

- Primários: mortalidade e desabilidade aos 18 meses. Houve redução significativa na combinação entre ambos: RR 0.76, a: 95\%, intervalo de confiança de 0.69 a $0.84, p<0.001$.

- Secundários: os mesmos componentes do desfecho primário, incluindo sobrevida com função neurológica normal (definida como: ausência de paralisia cerebral, no máximo $1 \mathrm{DP}$ abaixo das médias nos testes, visão e audição normais). Houve aumento da taxa de sobrevida com função neurológica normal: RR 1.63, a: 95\%, intervalo de confiança de 1.36 a 1.95, $p<0.001$. Avaliando o desfecho em relação à gravidade da encefalopatia, encontrou-se redução de risco na moderada e na severa, respectivamente: RR 0.67, a: 95\%, intervalo de confiança de 0.56 a 0.81 com NNT: 6 e RR 0.83, a: $95 \%$, intervalo de confiança de 0.74 a 0.92 com NNT: 7 . Também foi analisada a severidade da encefalopatia e método de hipotermia com relação ao desfecho. Independentemente do método utilizado, houve redução do risco de morte ou desabilidade: RR 0.75 , a: $95 \%$, intervalo de confiança de 0,66 a 0.85 no resfriamento corporal total e RR 0.77, a: $95 \%$, intervalo de confiança de 0,65 a 0.93 no resfriamento seletivo. 
Tais resultados permitem concluir que a hipotermia melhora a sobrevida e o desenvolvimento neurológico tanto nos bebês com encefalopatia moderada quanto nos com severa. Qualquer modalidade, seja seletiva ou total, é eficiente. E o mais importante, estabelece finalmente a necessidade de tornar a hipotermia uma terapêutica de rotina no cuidado desses RNs.

\section{Questões que ainda não estão bem respondidas}

Apesar de toda evidência científica, alguns questionamentos ainda aguardam resposta. A primeira questão é se haveria necessidade, como critério de inclusão, da realização de EEG. Embora sua realização seja recomendável, aliado ao exame neurológico, a fim de refinar a classificação de risco ${ }^{28}$, parece não ser necessário uma vez que o NICHD Trial não o utilizou e, mesmo assim, foi capaz de identificar - através de critérios clínicos e metabólicos - $62 \%$ dos pacientes com desfechos desfavoráveis, número semelhante ao do Cool Cap Trial $(66 \%)$ e do TOBY Trial $(53 \%)^{29}$.

Outra questão importante é em relação a possíveis efeitos adversos da hipotermia. Nesse sentido, ela parece não ter influenciado a ocorrência de infecção, pois a incidência de sepse, nas crianças submetidas à hipotermia e nos controles, variou de maneira semelhante, entre 2 e $12 \%$. Outra preocupação relevante se relacionava à possibilidade de aumentar o risco de sangramento mas embora tenha sido observada uma tendência maior nas crianças submetidas à terapia com hipotermia, não ocorreram complicações maiores quando comparadas a crianças normotérmicas ${ }^{29}$.

Algumas questões ainda não puderam ser respondidas e necessitam estudos específicos. A principal delas diz respeito ao uso da hipotermia em crianças com menor IG ou naqueles com estágio 1 de Sarnat, além do esclarecimento se 0 estabelecimento precoce do procedimento traria um maior benefício aos pacientes.

\section{Considerações Finais}

Em muitos países, hospitais tem introduzido a hipotermia para tratamento de RNs com EHI. Em 2010, tal tratamento recebeu aprovação do National Institute for Health and Clinical Excellence, do Reino Unido, bem como do International Liaison Committee on Resuscitation Guidelines. No Brasil, o procedimento é recomendado pela Sociedade Brasileira de Pediatria desde 2012. Até o momento não há publicações nacionais sobre o assunto.

Ao surgir uma nova terapia para determinada patologia, os profissionais da área procuram informar-se sobre o assunto; mais relevante ainda quando esta terapia é a única disponível para uma condição grave. Por isso, essa revisão se propôs a reunir os dados mais importantes e recentes sobre a hipotermia terapêutica, permitindo concluir que a mesma não só é segura, mas também eficaz em termos de redução da mortalidade e da ocorrência de desabilidades, nesta população específica.

Essa revisão que se propôs a reunir os dados mais importantes e recentes sobre a hipotermia terapêutica, permitiu concluir que a mesma não só é segura, mas também eficaz em termos de redução da mortalidade e da ocorrência de desabilidades, nesta população específica. Assim, os profissionais da área podem, através deste artigo, obter informações sobre esta terapia, que se torna mais relevante ainda quando ela é a única disponível para uma patologia que na maioria das vezes leva a condições clínica e consequência graves tanto para a criança como para a família.

\section{Referências Bibliográficas}

1. Fonseca LF, Xavier C, Pianetti G. Compêndio de Neurologia Infantil. 2a ed. Rio de Janeiro: Medbook; 2011.

2. Diament A, Cypel S, Reed UC. Neurologia Infantil. 5a ed. São Paulo: Atheneu; 2010.

3. Sarnat HB, Sarnat MS. Neonatal encephalopathy following fetal distress. A clinical and electroencephalographic study. Arch Neurol. 1976 Oct;33(10):696-705.

4. Jacobs SE, Berg M, Hunt R, Tarnow-Mordi WO, Inder TE, Davis PG. Cooling for newborns with hypoxic ischaemic encephalopathy. Cochrane Database Syst Rev. 2013 Jan;1:CD003311.

5. Shankaran S, Laptook AR, Ehrenkranz R a, Tyson JE, McDonald S a, Donovan EF, et al. Whole-body hypothermia for neonates with hypoxic-ischemic encephalopathy. N Engl J Med. 2005 Oct 13;353(15):1574-84.

6. Ministério da Saúde (Brasil), Coordenação Geral de Informação e Análise Epidemiológica da Secretaria de Vigilância em Saúde. Mortalidade infantil no Brasil: tendências, componentes e causas de morte no período de 2000 a 2010. Brasilia: Ministério da Saúde; 2011. 
7. Roth SC, Baudin J, Cady E, Johal K, Townsend JP, Wyatt JS, et al. Relation of deranged neonatal cerebral oxidative metabolism with neurodevelopmental outcome and head circumference at 4 years. Dev Med Child Neurol. 1997 Nov;39(11):718-25.

8. Roth SC, Edwards AD, Cady EB, Delpy DT, Wyatt JS, Azzopardi D, et al. Relation between cerebral oxidative metabolism following birth asphyxia, and neurodevelopmental outcome and brain growth at one year. Dev Med Child Neurol. 1992 Apr;34(4):285-95.

9. Edwards AD, Yue X, Squier M V, Thoresen M, Cady EB, Penrice J, et al. Specific inhibition of apoptosis after cerebral hypoxia-ischaemia by moderate post-insult hypothermia. Biochem Biophys Res Commun. 1995 Dec 26;217(3):1193-9.

10. Globus MY, Alonso O, Dietrich WD, Busto R, Ginsberg MD. Glutamate release and free radical production following brain injury: effects of posttraumatic hypothermia. J Neurochem. 1995 Oct;65(4):1704-11.

11. Edwards a D, Brocklehurst P, Gunn a. J, Halliday H, Juszczak E, Levene M, et al. Neurological outcomes at 18 months of age after moderate hypothermia for perinatal hypoxic ischaemic encephalopathy: synthesis and meta-analysis of trial data. BMJ. 2010 Feb 9;340(feb09 3):c363-c363.

12. Jacobs SE, Morley CJ, Inder TE, Stewart MJ, Smith KR, McNamara PJ, et al. Whole-body hypothermia for term and near-term newborns with hypoxic-ischemic encephalopathy: a randomized controlled trial. Arch Pediatr Adolesc Med. 2011 Aug;165(8):692-700.

13. Tagin M a, Woolcott CG, Vincer MJ, Whyte RK, Stinson D a. Hypothermia for neonatal hypoxic ischemic encephalopathy: an updated systematic review and meta-analysis. Arch Pediatr Adolesc Med. 2012 Jun 1;166(6):558-66.

14. Simbruner G, Mittal R a, Rohlmann F, Muche R. Systemic hypothermia after neonatal encephalopathy: outcomes of neo.nEURO.network RCT. Pediatrics. 2010 Oct;126(4):e771-8.

15. Zhou W, Cheng G, Shao X, Liu X, Shan R, Zhuang D, et al. Selective head cooling with mild systemic hypothermia after neonatal hypoxic-ischemic encephalopathy: a multicenter randomized controlled trial in China. J Pediatr. 2010 Sep;157(3):367-72, 372.e1-3.

16. Gluckman PD, Williams CE. When and why do brain cells die? Dev Med Child Neurol. 1992 Nov;34(11):1010-4.

17. Lorek A, Takei Y, Cady EB, Wyatt JS, Penrice J, Edwards AD, et al. Delayed ("secondary") cerebral energy failure after acute hypoxia-ischemia in the newborn piglet: continuous 48-hour studies by phosphorus magnetic resonance spectroscopy. Pediatr Res. 1994 Dec;36(6):699-706.

18. Penrice J, Cady EB, Lorek A, Wylezinska M, Amess PN, Aldridge RF, et al. Proton magnetic resonance spectroscopy of the brain in normal preterm and term infants, and early changes after perinatal hypoxia-ischemia. Pediatr Res. 1996 Jul;40(1):6-14. 19. Williams CE, Gunn A, Gluckman PD. Time course of intracellular edema and epileptiform activity following prenatal cerebral ischemia in sheep. Stroke. 1991 Apr;22(4):516-21.

20. Inder TE, Volpe JJ. Mechanisms of perinatal brain injury. Semin Neonatol. 2000 Feb;5(1):3-16.

21. Kida H, Nomura S, Shinoyama M, Ideguchi M, Owada Y, Suzuki M. The Effect of Hypothermia Therapy on Cortical Laminar Disruption following Ischemic Injury in Neonatal Mice. PloS one. 2013 Jan;8(7):e68877.

22. Laptook AR, Corbett RJ, Sterett R, Burns DK, Tollefsbol G, Garcia D. Modest hypothermia provides partial neuroprotection for ischemic neonatal brain. Pediatr Res. 1994 Apr;35(4 Pt 1):436-42.

23. Laptook AR, Corbett RJ, Sterett R, Burns DK, Garcia D, Tollefsbol G. Modest hypothermia provides partial neuroprotection when used for immediate resuscitation after brain ischemia. Pediatr Res. 1997 Jul;42(1):17-23.

24. Thoresen M, Penrice J, Lorek A, Cady EB, Wylezinska M, Kirkbride V, et al. Mild hypothermia after severe transient hypoxia-ischemia ameliorates delayed cerebral energy failure in the newborn piglet. Pediatr Res. 1995 May;37(5):667-70. 25. Van Leeuwen GM, Hand JW, Lagendijk JJ, Azzopardi D V, Edwards AD. Numerical modeling of temperature distributions within the neonatal head. Pediatr Res. 2000 Sep;48(3):351-6.

26. Gluckman PD, Wyatt JS, Azzopardi D, Ballard R, Edwards a D, Ferriero DM, et al. Selective head cooling with mild systemic hypothermia after neonatal encephalopathy: multicentre randomised trial. Lancet. 2005;365(9460):663-70.

27. Azzopardi D V, Strohm B, Edwards a D, Dyet L, Halliday HL, Juszczak E, et al. Moderate hypothermia to treat perinatal asphyxial encephalopathy. N Engl J Med. 2009 Oct 1;361(14):1349-58.

28. Shalak LF, LaptookAR, Velaphi SC, Perlman JM. Amplitude-integrated electroencephalography coupled with an early neurologic examination enhances prediction of term infants at risk for persistent encephalopathy. Pediatrics. 2003 Feb;111(2):351-7.

29. Thoresen M. Hypothermia after perinatal asphyxia: selection for treatment and cooling protocol. J Pediatr. Mosby, Inc.; 2011 Feb;158(2 Suppl):e45-9. 


\section{Raquel Trautenmüller Kerber Binkowski}

Endereço para correspondência - Avenida Júlio de Castilhos, $n^{\circ} 591$ - Sala 112. Bairro Centro, CEP: 98600-000, Três Passos, RS, Brasil.

E-mail: rtkerber@yahoo.com.br

Lattes: http://lattes.cnpq.br/3148450094551245

Angela Regina Maciel Weinmann - noca@rocketmail.com

Enviado em 10 de setembro de 2013. Aceito em 15 de dezembro de 2014. 
